\title{
Analyzing and detecting hemorrhagic and ischemic stroke- based on bit plane slicing and edge detection algorithms
}

\author{
Warqaa Shaher Alazawee, Zobeda Hatif Naji, Weaam Talaat Ali \\ Department of Computer Engineering, Faculty of Engineering Collage, University of Diyala, Diyala, Iraq
}

\begin{abstract}
Article Info
Article history:

Received Sep 30, 2021

Revised Dec 7, 2021

Accepted Dec 15, 2021

Keywords:

Bit plan slice transform

Brain stroke

CT images

Edge detection

ABSTRACT

Nowadays, in the medical world, analyzing and diagnosing acute brain stroke and its location is a difficult process. In many hospitals, however, striking symptoms with the use of computed tomography (CT) imaging for patients is an important step in screening and diagnosis. Furthermore, computer-assisted accurate detection of diseased brain regions Because of the several sorts of strokes, their uneven form, and their great intensity and size, aided design is extremely challenging. Using the bit plan slice technique and the canny detector, we created and suggested a novel approach. Our algorithm produces excellent outcomes. The results demonstrate that our proposed algorithm is an accurate and reliable technique. This study also indicates that this system can detect two different types of strokes: hemorrhagic and ischemic strokes. The results of a comparison study of our suggested technique and other methods such as negative and logarithmic transformation methods are also included in this article.
\end{abstract}

This is an open access article under the $\underline{C C B Y-S A}$ license.

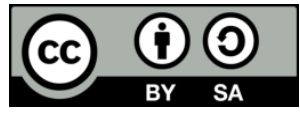

\section{Corresponding Author:}

Warqaa Shaher Alazawee

Department of Computer Engineering, Faculty of Engineering Collage, University of Diyala

Alkafaat St. Diyala, Baqubah, Iraq

Email: warqaash@uodiyala.edu.iq

\section{INTRODUCTION}

The brain has to be the most complicated part in the human body that serves as the nerve system's focal point [1]. It governs all bodily processes, therefore any illness or impairment to the brain will have a significant impact on human health, including motion and cognitive [2], [3]. When blood circulation carrying out food and oxygen to a section of brain tissue by channels (called arteries) is suddenly blocked, it is referred to as a stroke or a brain infarction. As a result, a portion of the brain does not receive the blood that its cells require. As a consequence of the shortage of sufficient blood supply, brain cells begin to deteriorate then die. Ischemic and hemorrhagic strokes are the two forms of stroke [2], [4], [5]. Thus, ischemic stroke occurs when the blood arteries in the brain get clogged, depriving brain tissue from receiving nutrients and oxygen. Blockages or narrowing of blood arteries in the brain were induced by fatty deposits that built up in the vessels [6]-[8]. Furthermore, recent evidence suggests that COVID-19 infection is linked to ischemic stroke [9]. While hemorrhagic stroke occurs when the blood vessels in the brain bleed or rupture. Stroke can be caused by a number of factors, including high blood pressure and excessive use of blood-thinning drugs [10]. The immediate cells in the area of the brain stroke are not the only ones affected. It also harms the cells in the surrounding area. The appearance, shape, size, and position of these two types of strokes are all distinct and different. These strokes were classified as either hypointense (darker than brain tissue) or hyperintense (brighter than brain tissue) [11], [12]. Because computed tomography (CT) scan pictures clearly reveal anything inside the skull, including bones, soft tissue, brains, as well as blood arteries, they will be the concentrate of this study [2]. Until date, CT scan has been the most appropriate mechanism of diagnosing 
brain strokes since it is available anytime and takes much less time than that of an magnetic resonance imaging (MRI) system [13]. CT scans employ spinning X-rays and computers to detect whether brain regions are attributed to a lack of blood (ischemic stroke) or a broken blood artery (hemorrhagic stroke). CT scan images may be shown as a brain Window that exposes bones for the brain [14]. There is also the Hounsfield unit, which depicts the hue for each section of the brain as depicted in the part of the brain. As a result, an ischemic stroke seems black because no blood is present, but a hemorrhagic stroke resembles brilliant because it is caused by a broken blood vessel [4], [10].

Several methodologies have been developed to meet the need for accurate detection and right analyzing ways to address brain stroke in CT images for use as a tool for diagnosing the stroke. Recently, Subudhi et al. (2020) studied segmentation and classification of brain stroke using expectation maximization and random forest classifiers. In their research, a diffusion-weighted image (DWT) has been found to detect the ischemic stroke. Their result showed that the algorithm is more efficient than the usual segmentation method. However, its computation is complex, and their algorithm works on just one type of stroke which is ischemic stroke [15]. Also, Zhang et al. (2020) studied ischemic stroke lesion segmentation using multi-plane information fusion. Their algorithm involved various steps for diagnosis of ischemic stroke. They designed DSN which is a detection and segmentation network. Then they proposed a multi-plane fusion network (MPFN). Their study showed that their algorithm is helpful for lesion segmentation, but it Also works on ischemic stroke, and it takes a long time [16]. Clerigues et al. (2019) proposed a method for acute ischemic stroke lesion core using full convolutional neural networks. Their result showed that the method can provide an estimate of lesion core size and location. However, they accompanied their method with other methods that have the same efficiencies with others [17]. Shi et al. (2019) studied modified U-Net architecture for ischemic stroke lesion segmentation and detection. Their Experiment results showed that their model can segment ischemic stroke, but they should choose suitable hyperparameters. In all, all these proposed methods are suffering from one or several disadvantages. This research, on the other hand, tries to provide an optimal categorization and detection strategy that would overcome these drawbacks. This suggested method also functions on both types of brain strokes [18].

Nowadays, it is undeniable that a more efficient approach of segmentation and boundary recognition that can be employed in real time is required. Therefore, the goal of this research is to analyze and discover a tool that can accurately recognize the stroke and its boundaries from CT scan images. As a result, this paper proposes a new strategy for segmentation and edge identification based on the bit plan slice transform method with the canny operator.

\section{PIECEWISE-LINEAR TRANSFORMATION}

Gray level transformation functions are one of the most important techniques in digital image processing. These techniques deal with pixel values. There are two principal categories of transformation techniques: which are intensity transformation techniques and spatial filtering techniques [19], [20]. The intensity transformation techniques operate on single pixels of an image. As a result, $\beta$ and $\delta$ will represent the value of each pixel before and after processing, and so these values will be associated by an equation of the form below:

$$
\beta \propto \delta \gg \beta=T(\delta)
$$

where $\mathrm{T}$ is a transformation function that maps pixel values of $\delta$ into pixel values of $\beta$. The spatial filtering techniques deal with operations that work on a neighborhood of every pixel in an image [19]. These operations can be denoted by the expression:

$$
G(x, y)=T[f(x, y)]
$$

where $\mathrm{T}$ is an operator that defined over a neighborhood, $G(x, y)$ represents the output image and $f(x, y)$ is the input image. These techniques can be classical techniques or fuzzy techniques. In this paper will focus on the intensity transformation techniques. The basic types of these techniques are (negative and identity transformation), logarithmic (log and inverse log transformation), and power-law transformation. All these techniques work on intensity of image [19]. However, in this research we deal with a complementary approach to all these methods above which are piecewise linear transformation functions. Contrast stretching, Gray-level slicing, and bit plane slicing are three types of piecewise linear transformation [19]. We proposed bit plane slicing to address the two types of brain stroke. Bit plane slicing is a function of representing an image used for each pixel. It highlights the image depending on specific and desired bit in pixel [21]. The focul procedure in this approach, which is made of numerous processes illustrated in Figure 1, was bit plane slicing and edge extraction. 


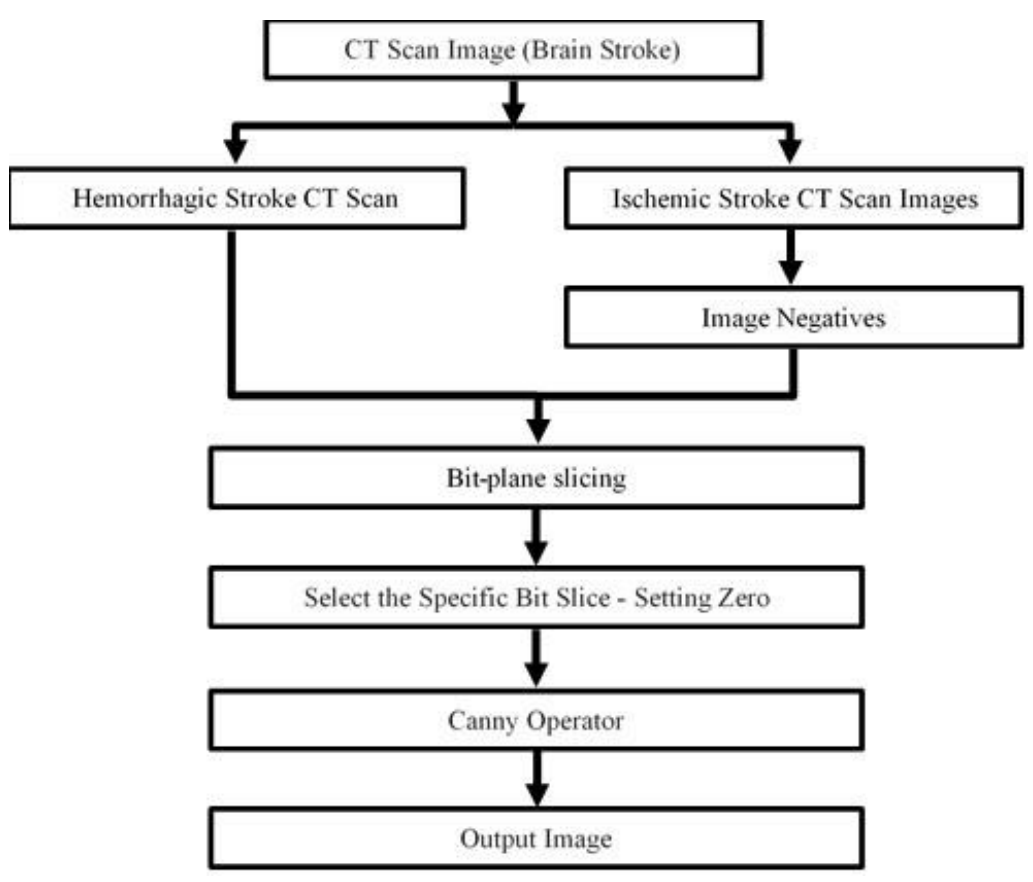

Figure 1. Proposed algorithm steps

\section{METHOD}

In this section a full detail of the proposed methode will be described in steps as:

Step 1: Image negatives

Image negative has been used to reverse the intensity levels of an image. In this manner produces the equivalent of photographic negative [19]. So, the negative transformation for an image with gray levels in the range $[0, \mathrm{~L}-1]$ is given by the expression:

$$
S=L-l-r
$$

gray level image is inverted, each of its pixel value ' $r$ ' is subtracted from the maximum pixel value (L-1). Image inversion is suited for enhancing gray or white detail embedded in dark regions of an image when the dark areas to rev are dominant in size [19]. However, in our work image inversion is employed to work as pre-processing for ischemic stroke images.

Ischemic stroke appears as a dark area in CT scan images. So, this type of transformation has been used to invert ischemic stroke images to bright areas rather than dark areas before applying our algorithm. That will be much easier to analyze the brain tissue in the negative images.

Step 2: Bit -plane slicing

In medical images applications include dealing with specific areas and features that are desired to highlight a specific range of gray levels in an image [22]. Level slicing plays a vital role in this field. There are several ways of doing it. However, most of them are variations of two themes. One approach based on displaying high value for all gray levels of interest areas, while a low value displays all other gray levels. The second approach based on brightens the interest range of gray levels and preserves the other gray level as background in image [19], [21].

In this paper, bit plane slicing has been used, in this method can present the total image by specific bits. As known, each pixel in an image is presented by 8 bits. So, the image is composed of eight 1-bit planes, ranging from the bit- plane for the least significant bit (0) to the most significant bit (7). Plane (0) contains all the lowest order bits, while plane (7) contains all high-order bits that include all most visually significant data as shown in Figure 2 [19].

In our work, we employ bit plane slicing to separate the CT images for ischemic and hemorrhagic stroke images into its bits planes to analyze and discard the levels that do not contribute to identifying the brain stroke. Thus, we will detect the bit slices that contain all beneficial information about the stroke. In setting specific bits to zero, we maintain the bit slices that contain the most useful information and appear the texture of the stroke clearly that can be used for features extraction to classify the stroke and detect the sharpened edge. 


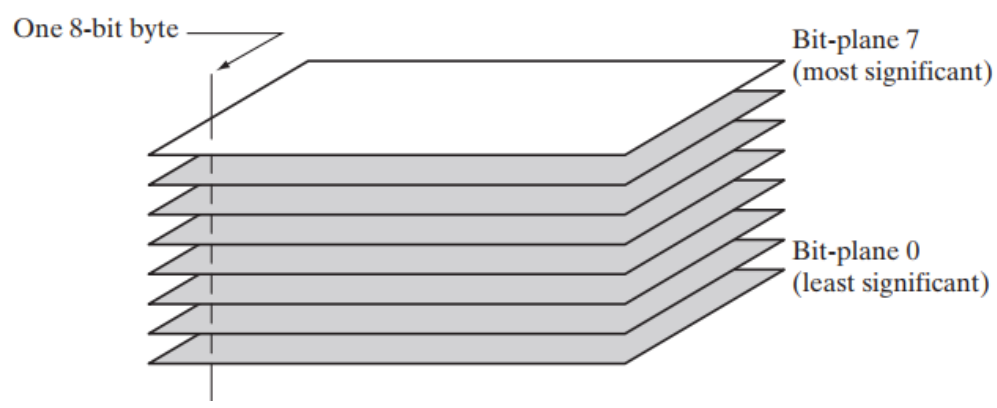

Figure 2. Bit-plan slicing

Step 3: Edge extraction

For medical images, edge extraction is a critical approach. It's described as the process of detecting and finding acute discontinuities, which are sudden changes in pixel intensity that define the borders of objects in a picture [23]. Gradient-based methods and laplacian-based methods [24] are two strategies for modeling edges [25], [26]. However, we suggested the canny operator as a more sophisticated approach for edge detection in our study. Although the canny edge detector is a complicated algorithm, it is the best. A sequence of steps are followed to implement the canny edge detection method [27]. Before recognizing any edges, canny first filters away any noise or disturbance in the original picture [19]. Gaussian filter has been used which is the simplest and easiest way to do that process as represented by (1) and (2).

$$
\begin{aligned}
& G(x, y)=e^{-\frac{x^{2}+y^{2}}{2 \sigma^{2}}} \\
& f_{S}(x, y)=G(x, y) * f(x, y)
\end{aligned}
$$

The image's gradient is used to determine the edge strength in the step. To execute a 2-D spatial gradient, the Sobel operator is used [18]. The edge direction is determined in the third step using the gradient in the $\mathrm{x}$ and $\mathrm{y}$ axes. The edge direction is determined by whether the gradient in the $\mathrm{x}$ and $\mathrm{y}$ directions is equal to zero or 90 degrees. Once determining the edge direction, this should link it to a direction that can be tracked in an image [19]. As an example, suppose the pixels in a 5x5 image are as given in Figure 3.

\begin{tabular}{|c|c|c|c|c|}
\hline $\mathbf{x}$ & $\mathbf{x}$ & $\mathbf{x}$ & $\mathbf{x}$ & $\mathbf{x}$ \\
\hline $\mathbf{x}$ & $\mathbf{x}$ & $\mathbf{x}$ & $\mathbf{x}$ & $\mathbf{x}$ \\
\hline $\mathbf{x}$ & $\mathbf{x}$ & $\mathbf{a}$ & $\mathbf{x}$ & $\mathbf{x}$ \\
\hline $\mathbf{x}$ & $\mathbf{x}$ & $\mathbf{x}$ & $\mathbf{x}$ & $\mathbf{x}$ \\
\hline $\mathbf{x}$ & $\mathbf{x}$ & $\mathbf{x}$ & $\mathbf{x}$ & $\mathbf{x}$ \\
\hline
\end{tabular}

Figure 3. An example of an image of $5 \times 5$ pixsels

The four available orientations for characterizing the surrounding pixels are $0^{\circ}, 45^{\circ}, 90^{\circ}$, and $135^{\circ}$. As a result, depending on whatever direction the edge is nearest to, it must be resolved into one of these four directions. Non-maximum suppression must be used in the following stage. It's used to trace along the edge along the direction of the edge. Then, every pixel value that isn't deemed an edge is set to zero. This step will aid in the creation of a narrow line in the final image [19]. Finally, the canny edge method employs hysteresis. This procedure is used to get rid of streaks. The breaking apart of an edge contour produced by the operator output varying above and below the threshold is known as streaking. This stage determines whether edges are indeed edges, and which aren't. Two threshold values are utilized for this: a minimum value (T1) and a maximum value (T2). So, the edges with intensity gradient more than maximum value are sure to be edges, while all other edges below minimum value are discarded because they are sure to be non-edges. Moreover, all other edges who lie between these two thresholds are classified edges. If they are connected to "sure-edge" pixels. Otherwise, other edges are also discarded [24]. Because of the necessity for more effective ways for detecting edges of a brain stroke, we utilized this advanced edge detection algorithm in our study. This can aid in the correct analysis and identification of a brain stroke accurately. 


\section{RESULTS AND DISCUSSION}

Real data of hemorrhagic and ischemic stroke pictures were used to validate our suggested technique. The Baqubah Teaching Hospital has provided several images. To compare and assess the performance of our algorithms, we utilized the same data using the following transformation techniques: negative, and logarithmic.

\subsection{Ischemic stroke results}

Figure 4(a) displays the original image of ischemic stroke Figure 4(b) shows the obtained image after applying image negatives that will be so useful before applying our proposed method because it makes the area of ischemic stroke brighter than its area in the original image. Figure 4(c) shows the eighth bit slices of ischemic stroke. In this step, our method separates each bit level into an image that can help in detecting the levels that contribute to identifying the stroke and discarding the other levels. Figure 4(d) shows the step of Setting specific bits to zero. We set the bits1,2,3,4,8 to zero and maintain only bits 5,6,7, where most of the information is included. As can be seen from Figure 4(d) the $7^{\text {th }}$ bit image. has wide variance of intensities and includes all information about ischemic stroke, while the $5^{\text {th }}$ and $6^{\text {th }}$ slices were considered as supporting information. So, we crop the area of ischemic stroke that is shown in the $7^{\text {th }}$ bit image. Figure 4(e) shows the result after applying a canny edge detector. The result shows the canny operator detects the precise boundary of ischemic stroke area by providing low error edges, continuous edges, and edges with one pixel wide.

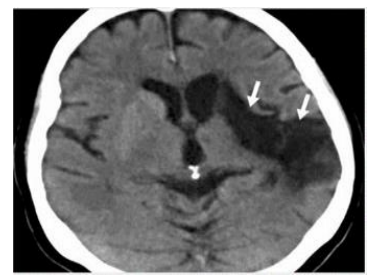

(a)

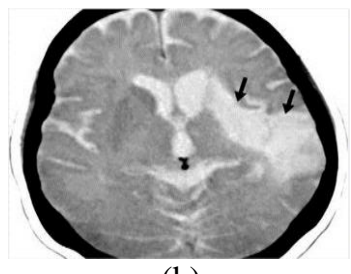

(b)
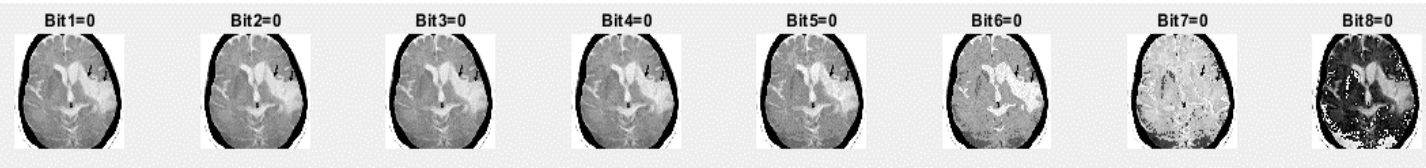

(c)
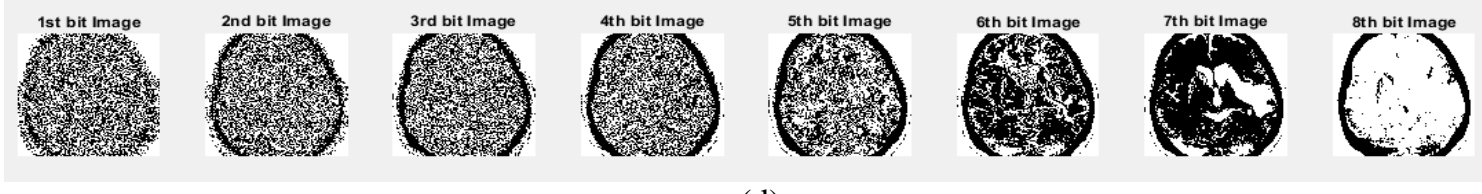

(d)

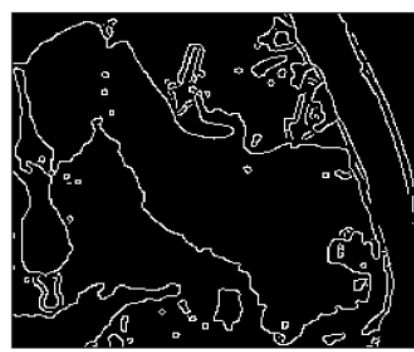

(e)

Figure 4. Ischemic stroke results extraction and detection: (a) original image (ischemic stroke), (b) negative image (ischemic stroke), (c) bit-plane slicing images, (d) setting specific bits to zero (Ischemic stroke), and

(e) edges of (ischemic stroke) by canny detector

\subsection{Hemorrhagic stroke results}

The initial image of a hemorrhagic stroke is shown in Figure 5(a). This type of stroke does not need to apply a negative method before applying our proposed algorithm because the area of hemorrhagic stroke is 
brighter than other tissue in the CT scan images. After performing bit-plane slicing, the result of hemorrhagic stroke is shown in Figure 5(b). For hemorrhagic stroke also, the output shows the most significant bit slices which $\left(5^{\text {th }}, 6^{\text {th }}, 7^{\text {th }}\right)$ include all important information and features. Figure $5(\mathrm{c})$ shows the result after setting bits $1,2,3,4,8$ to zero and keeping the $5^{\text {th }}, 6^{\text {th }}$, and $7^{\text {th }}$ bits $(5,6,7)$. Also, we crop the area of the hemorrhagic stroke in the seven-bit slice that contains all information about the stroke. Figure 5(d) shows the result using a canny edge detector. Also, canny edge detector detects the area of hemorrhagic stroke accurately. It provides continuous edges with clear localization, and thin edges.

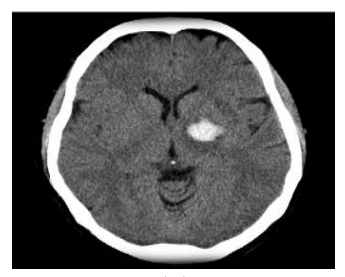

(a)
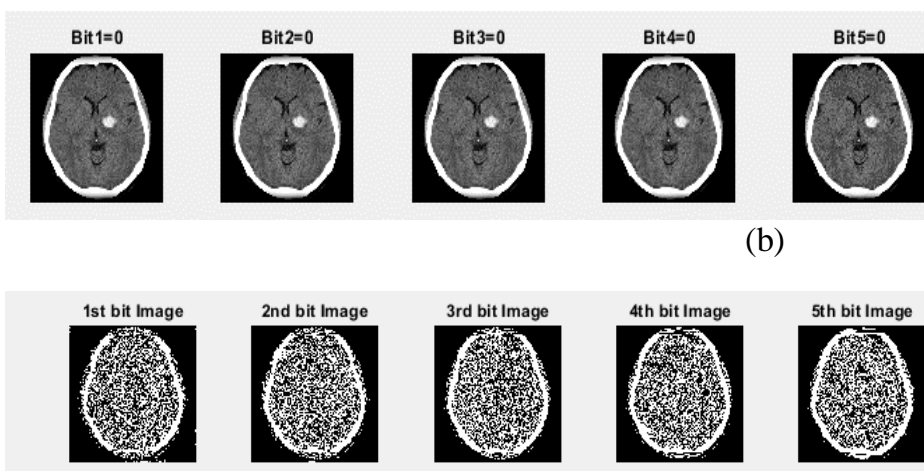

(c)

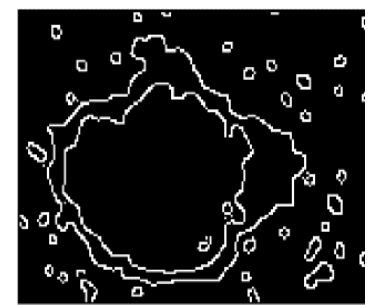

(d)

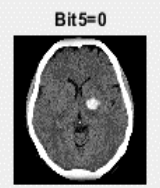

(b)
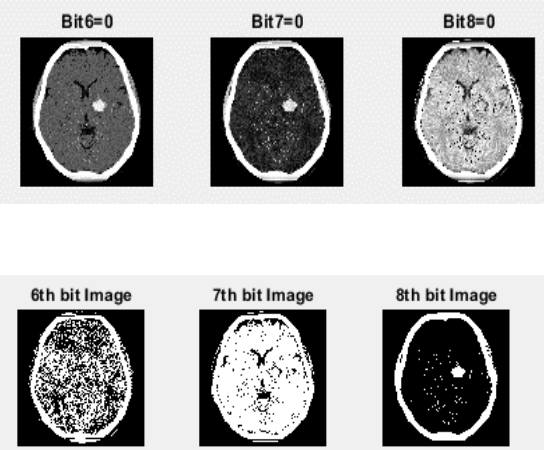

Figure 5. Hemorrhagic stroke results extraction and detection: (a) original image (hemorrhagic stroke),

(b) bit plane slicing for (hemorrhagic stroke), (c) setting specific bits to zero (hemorrhagic stroke), and

(d) edges of (hemorrhagic stroke) by canny detector

\subsection{Negative, and logarithmic image results}

Figures 6(a)-(j) show the results after applying different methods which are negative and logarithmic to compare our proposed method with these two methods. The initial image of an ischemic stroke is shown in Figure 6(a). Figure 6(b) illustrates the effect of applying the negative approach to an image of an ischemic stroke. The outcome of applying the canny operator on negative ischemic stroke pictures is shown in Figure 6(c). Figure 6(d) shows the original image of hemorrhagic stroke image. Figure 6(e) illustrates the effect of applying the negative approach to the image of a hemorrhagic stroke. The outcome of using the canny operator on a negative hemorrhagic stroke picture is shown in Figure 6(f). The output of using the logarithmic approach for ischemic is shown in Figure $6(\mathrm{~g})$. The result of the canny operator for logarithmic ischemic stroke is shown in Figure 6(h). The outcome of using the logarithmic approach for the hemorrhagic stroke picture is shown in Figure 6(i) The output of using the canny operator on a logarithmic hemorrhagic stroke picture is shown in Figure 6(j). As can be seen from Figures 6(a)-(j) these two intensity transformation techniques do not work very well at analyzing and detecting areas of stroke in images. The area of stroke is unclear. Also, canny edge detector does not work very well with these two methods because these two methods cannot identify the area of stroke precisely. 


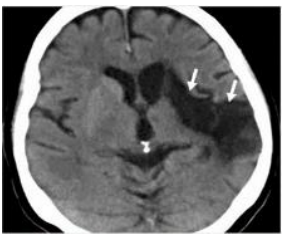

(a)

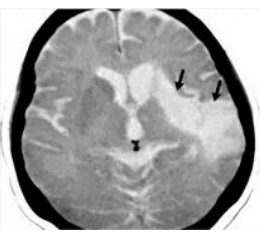

(b)

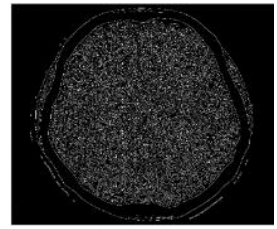

(f)

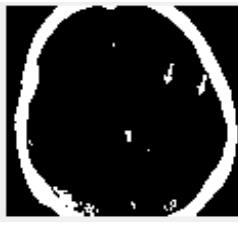

(g)

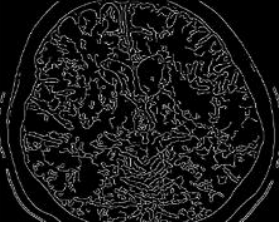

(c)

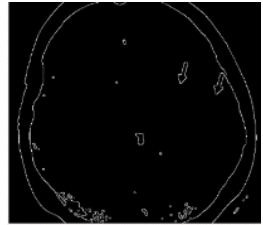

(h)

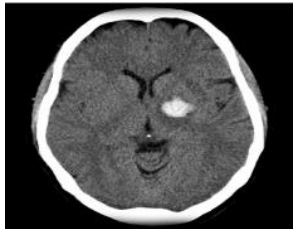

(d)

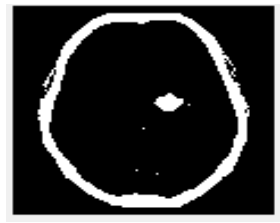

(i)

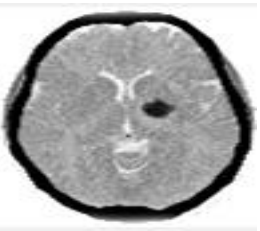

(e)

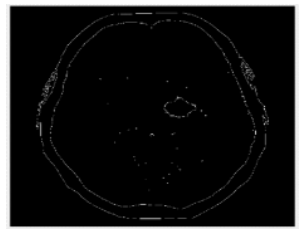

(j)

Figure 6. Negative, and logarithmic image results extraction and detection: (a) original image (ischemic stroke), (b) negative image (ischemic stroke), (c) canny for negative image (ischemic stroke), (d) original

image (hemorrhagic stroke), (e) negative image (hemorrhagic stroke), (f) canny for negative image

(hemorrhagic stroke), (g) logarithm image (ischemic stroke), (h) canny for logarithm image (ischemic stroke), (i) logarithm image (hemorrhagic stroke), and (j) canny for logarithm image (hemorrhagic stroke)

\section{CONCLUSION}

We designed and implemented a novel segmentation and edge detection approach that uses bit-plane slicing and the canny edge detector to isolate, address, and identify the border of a brain stroke region in this paper. As has been demonstrated, our suggested strategy produces outstanding accuracy outcomes. Negative and logarithmic approaches have also been implemented in order to conduct a comparative research between the suggested method in this study and these methods. Our suggested technique successfully identified and localized the region of interest, namely the brain stroke site, from other brain tissue. Our method creates clean, thin, and continuous edges while being time efficient. Furthermore, the system was tested on a variety of photos depicting various forms of brain stroke. More testing will be done to confirm the usage of this technology for different sorts of medical images.

\section{REFERENCES}

[1] K. A. Abu Nawas, M. Mustafa, R. Samad, D. Pebrianti, and N. R. Hasma Abdullah, "K-NN Classification of Brain Dominance," Int. J. Electr. Comput. Eng., vol. 8, no. 4, p. 2494, Aug. 2018, doi: 10.11591/ijece.v8i4.pp2494-2502.

[2] A. Alamoudi and Y. Abdallah, "Characterization of Brain Stroke Using Image and Signal Processing Techniques," in Biomedical Signal and Image Processing, Y. Zhou, Ed. IntechOpen, 2021, doi: 10.5772/intechopen.96288.

[3] L.-C. Hung, S.-F. Sung, and Y.-H. Hu, "A Machine Learning Approach to Predicting Readmission or Mortality in Patients Hospitalized for Stroke or Transient Ischemic Attack," Appl. Sci., vol. 10, no. 18, 2020, doi: 10.3390/app10186337.

[4] A. F. F. Alves, "Image Processing for Enhancement of Ischemic Stroke in Computed Tomography Examinations," PhD diss., Université d'Orléans, p. 109, 2019.

[5] M. Rudiansyah, T. A. Sardjono, and R. Mardiyanto, "Segmentation of the Intracerebral Hemorrhagic Strokes (Bleeds) from Brain CT Image Based on GVF Snake," in 2018 International Seminar on Intelligent Technology and Its Applications (ISITIA), Bali, Indonesia, pp. 465-470, Aug. 2018, doi: 10.1109/ISITIA.2018.8711155.

[6] J. Zhang, F. Shi, L. Chen, Z. Xue, L. Zhang, and D. Qian, "Ischemic Stroke Segmentation from CT Perfusion Scans Using Cluster-Representation Learning," in Machine Learning in Clinical Neuroimaging and Radiogenomics in Neuro-oncology, Cham, pp. 67-76, 2020, doi: 10.1007/978-3-030-66843-3_7.

[7] G. Wang, T. Song, Q. Dong, M. Cui, N. Huang, and S. Zhang, "Automatic ischemic stroke lesion segmentation from computed tomography perfusion images by image synthesis and attention-based deep neural networks," Med. Image Anal., vol. 65, p. 101787, Oct. 2020, doi: 10.1016/j.media.2020.101787.

[8] R. M. Sarmento, F. F. X. Vasconcelos, P. P. R. Filho, W. Wu, and V. H. C. de Albuquerque, "Automatic Neuroimage Processing and Analysis in Stroke-A Systematic Review," IEEE Rev. Biomed. Eng., vol. 13, pp. 130-155, 2020, doi: 10.1109/RBME.2019.2934500.

[9] S. Zhang, S. Xu, L. Tan, H. Wang, and J. Meng, "Stroke Lesion Detection and Analysis in MRI Images Based on Deep Learning," J. Healthc. Eng., vol. 2021, pp. 1-9, Apr. 2021, doi: 10.1155/2021/5524769.

[10] Y. Xu et al., "Deep Learning-Enhanced Internet of Medical Things to Analyze Brain CT Scans of Hemorrhagic Stroke Patients: A New Approach,” IEEE Sens. J., vol. 21, no. 22, pp. 24941-24951, Nov. 2021, doi: 10.1109/JSEN.2020.3032897.

[11] R. Sathish, R. Rajan, A. Vupputuri, N. Ghosh, and D. Sheet, “Adversarially Trained Convolutional Neural Networks for Semantic Segmentation of Ischaemic Stroke Lesion using Multisequence Magnetic Resonance Imaging," in 2019 41st Annual International Conference of the IEEE Engineering in Medicine and Biology Society (EMBC), Berlin, Germany, Jul. 2019, pp. 1010-1013, doi: 10.1109/EMBC.2019.8857527.

[12] M. Yousefian, A. Amani, H. Seyedarabi, and M. Farhoudi, "A comparison study of automated approaches for brain lesions segmentation in ischemic stroke," in 2020 28th Iranian Conference on Electrical Engineering (ICEE), Tabriz, Iran, Aug. 2020, pp. 1-7, doi: 10.1109/ICEE50131.2020.9260774. 
[13] W. Alomoush, A. Alrosan, A. Almomani, K. Alissa, O. A. Khashan, and A. Al-nawasrah, "Spatial information of fuzzy clustering based mean best artificial bee colony algorithm for phantom brain image segmentation," Int. J. Electr. Comput. Eng., vol. 11, no. 5, p. 4050, Oct. 2021, doi: 10.11591/ijece.v11i5.pp4050-4058.

[14] Y. Abdallah, "Segmentation of Brain Stroke Lesions using Marker-based Algorithms in CT images," in 2020 3rd International Conference on Computer Applications \& Information Security (ICCAIS), Riyadh, Saudi Arabia, Mar. 2020, pp. 1-4, doi: 10.1109/ICCAIS48893.2020.9096866.

[15] A. Subudhi, M. Dash, and S. Sabut, "Automated segmentation and classification of brain stroke using expectation-maximization and random forest classifier," Biocybern. Biomed. Eng., vol. 40, no. 1, pp. 277-289, Jan. 2020, doi: 10.1016/j.bbe.2019.04.004.

[16] L. Zhang et al., "Ischemic Stroke Lesion Segmentation Using Multi-Plane Information Fusion," IEEE Access, vol. 8, pp. 45715-45725, 2020, doi: 10.1109/ACCESS.2020.2977415.

[17] A. Clèrigues, S. Valverde, J. Bernal, J. Freixenet, A. Oliver, and X. Lladó, “Acute ischemic stroke lesion core segmentation in CT perfusion images using fully convolutional neural networks," Comput. Biol. Med., vol. 115, p. 103487, Dec. 2019, doi: 10.1016/j.compbiomed.2019.103487.

[18] W. Shi and H. Liu, "Modified U-Net Architecture for Ischemic Stroke Lesion Segmentation and Detection," in 2019 IEEE 4th Advanced Information Technology, Electronic and Automation Control Conference (IAEAC), Chengdu, China, Dec. 2019, pp. 1068-1071, doi: 10.1109/IAEAC47372.2019.8997642.

[19] R. C. Gonzalez, R. E. Woods, and B. R. Masters, "Digital Image Processing, Third Edition," J. Biomed. Opt., vol. 14, no. 2, p. 029901, 2009, doi: 10.1117/1.3115362.

[20] Z. H. Naji, "Image Segmentation Using Discrete Wavelets Transform,” Diyala J. Eng. Sci., vol. 13, no. 3, pp. 1-8, Sep. 2020, doi: $10.24237 /$ djes.2020.13301.

[21] H. K. Albahadily, V. Y. Tsviatkou, and V. K. Kanapelka, "Grayscale Image Compression using Bit Plane Slicing and Developed RLE Algorithms," Int. J. Adv. Res. Comput. Commun. Eng., vol. 6, no. 2, p. 6, 2017.

[22] A. Makandar and R. Somshekhar, “Alzheimer's disease detection using bit plane slicing Images," vol. 6, no. 3, p. 7, 2018.

[23] S. Kumar, A. K. Upadhyay, P. Dubey, and S. Varshney, "Comparative analysis for Edge Detection Techniques," in 2021 International Conference on Computing, Communication, and Intelligent Systems (ICCCIS), Greater Noida, India, Feb. 2021, pp. 675-681, doi: 10.1109/ICCCIS51004.2021.9397225.

[24] W. S. AlAzawee, I. Abdel-Qader, and J. Abdel-Qader, "Using morphological operations \&\#x2014; Erosion based algorithm for edge detection," in 2015 IEEE International Conference on Electro/Information Technology (EIT), Dekalb, IL, USA, May 2015, pp. 521-525, doi: 10.1109/EIT.2015.7293391.

[25] B. N. Shah and J. K. Bhalani, "Comparative analysis and implementation of structured Edge Active Contour," Int. J. Electr. Comput. Eng., vol. 10, no. 2, p. 1842, Apr. 2020, doi: 10.11591/ijece.v10i2.pp1842-1848.

[26] Z. Hussain and D. Agarwal, "A Comparative Analysis Of Edge Detection Techniques Used In Flame Image Processing," International Journal of Advance Research In Science And Engineering IJARSE, vol. 4, p. 10, 2015.

[27] R. V. Kumar Reddy, K. P. Raju, M. J. Kumar, L. R. Kumar, P. R. Prakash, and S. S. Kumar, "Comparative Analysis of common Edge Detection Algorithms using Pre-processing Technique,” Int. J. Electr. Comput. Eng., vol. 7, no. 5, p. 2574, Oct. 2017, doi: 10.11591/ijece.v7i5.pp2574-2580.

\section{BIOGRAPHIES OF AUTHORS}

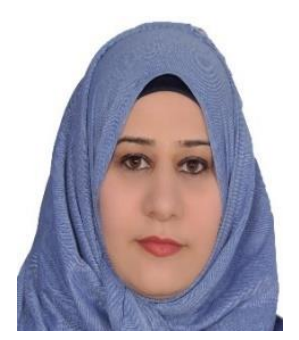

Warqaa Shaher Alazawee (iD) SC SC has received a bachelor's degree in computer engineering form University of Diyala /Iraq in 2007 and then worked as an Engineer in the University of Diyala/College of engineering from 2008 until the year 2012 and then she obtained a scholarship to get a master's degree from the United State of America which has been awarded in 2015 in computer engineering from Western Michigan University in United State of America. After that, she worked as a lecturer in the University of Diyala/ College of engineering until now. During this period, she published in journals and international conferences. Her research interests are focused on: (Computer Architecture, Image processing, medical image analysis, Biomedical Signal Processing). She can be contacted at email: warqaash@uodiyala.edu.iq and warqaashaher@gmail.com.

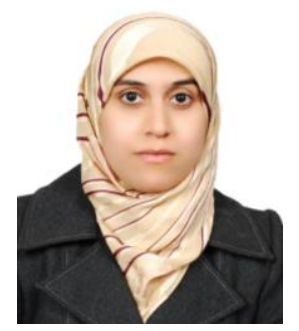

Zobeda Hatif Naji (D) SC P Ms. C. Electrical and Electronic Engineering 2017 MIPT institution in Russia. B.Sc. computer and software Engineering 2007 college of engineering Diyala university, Training, and course: Computer and Internet in Diyala University 2018. Methods of Teaching Course for one month in College of Education Diyala University, Iraq 2017. Test the safety of the Arabic language in Diyala University 2018. Teaching validity test in Diyala University 2018. She can be contacted at email: zobedahatifnaji@ uodiyala.edu.iq.

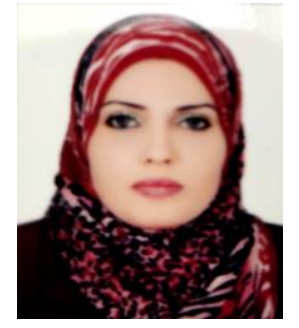

Weaam Talaat Ali (iD SC SC was born in Baghdad -Iraq 1984. She completed. Her bachelor's degree from Electric Engineering department Almustansiriya. University- College of Engineering, Iraq 2006, master's (Electronic and Communication) from Almustansiriya University, Iraq in 2012. Currently, she is a Lecturer in the Department of Computer Engineering, University of Diyala, Diyala, Iraq. Her research interests are Wireless communication, Signal and Image Processing, and system security. She can be contacted at email: wface2010@yahoo.com. 